\section{Un engramme ocytocinergique pour apprendre et contrôler sa peur}

Angel Baudon, Alexandre Charlet
CNRS et université de Strasbourg, institut des neurosciences cellulaires et intégratives (INCI, UPR3212), 8 allée du Général Rouvillois, 67000 Strasbourg, France. acharlet@unistra.fr

\section{Mémoire et engramme}

La mémoire est un processus permettant aux êtres vivants de stocker des informations afin de mieux s'adapter à leur environnement. L'idée d'une représentation physique de la mémoire remonte à plus de 2000 ans. En 350 avant J.-C., Aristote théorisait que «le processus de stimulation sensorielle marque une sorte d'impression du percept, juste comme un sceau laisse son empreinte dans une cire chaude »[1]. Cette idée a progressivement conduit à l'hypothèse selon laquelle des ensembles de cellules, organisés et sélectivement activés, forment les blocs de base de la trace mnésique, l'engramme. Cette hypothèse constitue aujourd'hui un champ majeur d'investigations, guidant l'étude des mécanismes cellulaires et moléculaires sousjacents à l'encodage et la préservation de la mémoire, qui restent pourtant encore mal connus. Ainsi, les informations seraient encodées par l'activité de micro-circuits précis au sein d'un réseau de neurones, de la même manière qu'une constellation peut se dessiner dans la voûte céleste. Une équipe internationale animée et coordonnée par Alexandre Charlet (France) et Valery Grinevich (Allemagne) a récemment montré que des engrammes sont susceptibles de se former dans certaines régions du cerveau comme l'hypothalamus.

\section{Ocytocine et amygdale cérébrale}

Afin de mettre en évidence un tel engramme, Hasan et al. [2] se sont intéressés à la création d'un souvenir de peur associée au contexte chez le rat. Chez cette espèce, la peur peut se manifester par une réaction comportementale d'immobilité appelée pétrification. Cette prétrification est sous le contrôle de nombreuses structures, ou régions, du cerveau, dont l'amygdale, une structure sous-corticale au cœur de la modulation des émotions, et I'hypothalamus, une région apparue très tôt au cours de l'évolution, impliquée dans l'homéostasie et la modulation de l'équilibre émotionnel. L'activité électrique des neurones de l'amygdale est modulée par l'activité de nombreux neurotransmetteurs ou neuromodulateurs, dont le glutamate, l'acide $\gamma$-amino-butyrique (GABA), la vasopressine, et l'ocytocine [3].

L'ocytocine, découverte en 1906 par Sir Henry Dales [4], est un petit peptide de neuf acides aminés synthétisé presque exclusivement dans I'hypothalamus, plus particulièrement dans le noyau paraventriculaire. Ce neuropeptide est produit par de grands neurones dits magnocellulaires, capables de sécréter l'ocytocine dans le sang, et par de petits neurones dits parvocellulaires, ne libérant l'ocytocine que dans le système nerveux central [5]. L'ocytocine joue un rôle majeur dans le comportement prosocial en favorisant les interactions sociales, la confiance, l'empathie, le comportement parental ou, plus généralement, les interactions sociales [5]. Outre ces effets désormais bien décrits et très médiatisés, on lui reconnaît aujourd'hui d'importantes fonctions comme molécule analgésique
[6], anti-stress et anxiolytique, impliquant notamment sa libération axonale dans l'amygdale $[2,7]$. Ainsi, la libération d'ocytocine endogène dans l'amygdale (Figure 1A) induit une forte diminution de la réaction de peur conditionnée (pétrification) chez le rat, suggérant que le dialogue entre l'hypothalamus et l'amygdale pourrait être fortement impliqué dans le contrôle des comportements de peur.

Pourtant, comment expliquer qu'un neuropeptide synthétisé dans un seul noyau cérébral puisse posséder autant de fonctions complémentaires? Est-il possible d'isoler et de définir des sous-populations fonctionnelles de neurones ocytocinergiques? C'est ce questionnement qui a poussé les chercheurs à s'intéresser à ces ensembles de cellules hypothalamiques potentiellement impliqués.

\section{Un engramme ocytocinergique contrôlant la peur}

$\varepsilon n$ utilisant une combinaison de vecteurs viraux permettant l'identification des neurones activés durant une courte fenêtre temporelle, les chercheurs ont montré que seule une sous-population de neurones ocytocinergiques hypothalamiques ( 13\%) est active durant l'exposition à un stimulus induisant une réaction de peur chez le rat (Figure IB). Grâce au système viral utilisé, ils ont pu faire exprimer des récepteurs exogènes uniquement par ces neurones activés lors d'une peur, ce qui leur a permis de manipuler leur activité. L'utilisation d'outils optogénétiques et chimiogénétiques leur 

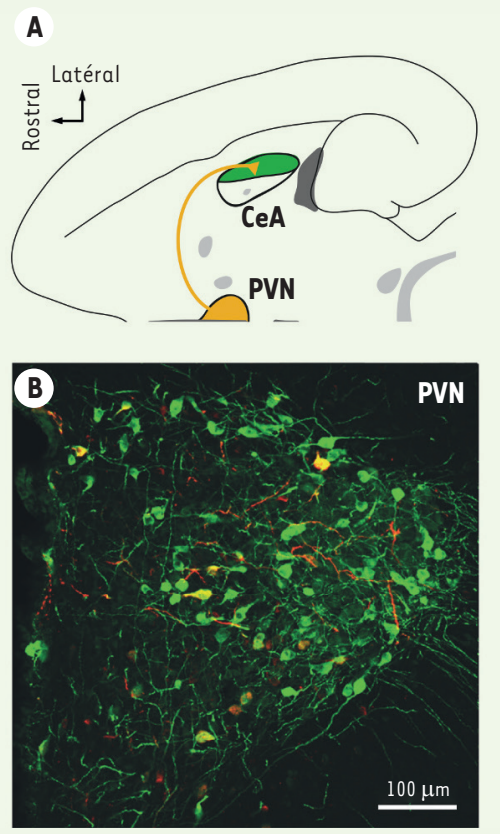

Figure 1. Identification des neurones activés lors d'une peur chez le rat. A. Les neurones ocytocinergiques du noyau paraventriculaire de I'hypothalamus (PVN; en orange) projettent leurs axones vers l'amygdale centrale (CeA; en vert). B. Seuls $13 \%$ des neurones ocytocinergiques sont activés par une expérience de peur. En vert: immunomarquage de l'ocytocine révélant tous les neurones produisant ce neuropeptide. En rouge : expression de la molécule fluorescente mCherry par les seuls neurones activés.

a ainsi permis de montrer que l'excitation spécifique de ce groupe de neurones écourte la durée de la pétrification, et qu'à l'inverse, leur inhibition augmente cette durée. Ainsi, ces quelques neurones ocytocinergiques sont à la fois nécessaires et suffisants pour contrôler la réaction de peur chez le rat (Figure 2) [2].

Cependant, les critères de nécessité et de suffisance dans la modulation d'une émotion par un groupe de neurones ne permettent pas d'établir l'existence d'un engramme. II est également important de montrer que ce groupe de neurones est recruté spécifiquement lors d'une émotion donnée. Aussi, les chercheurs ont comparé les populations

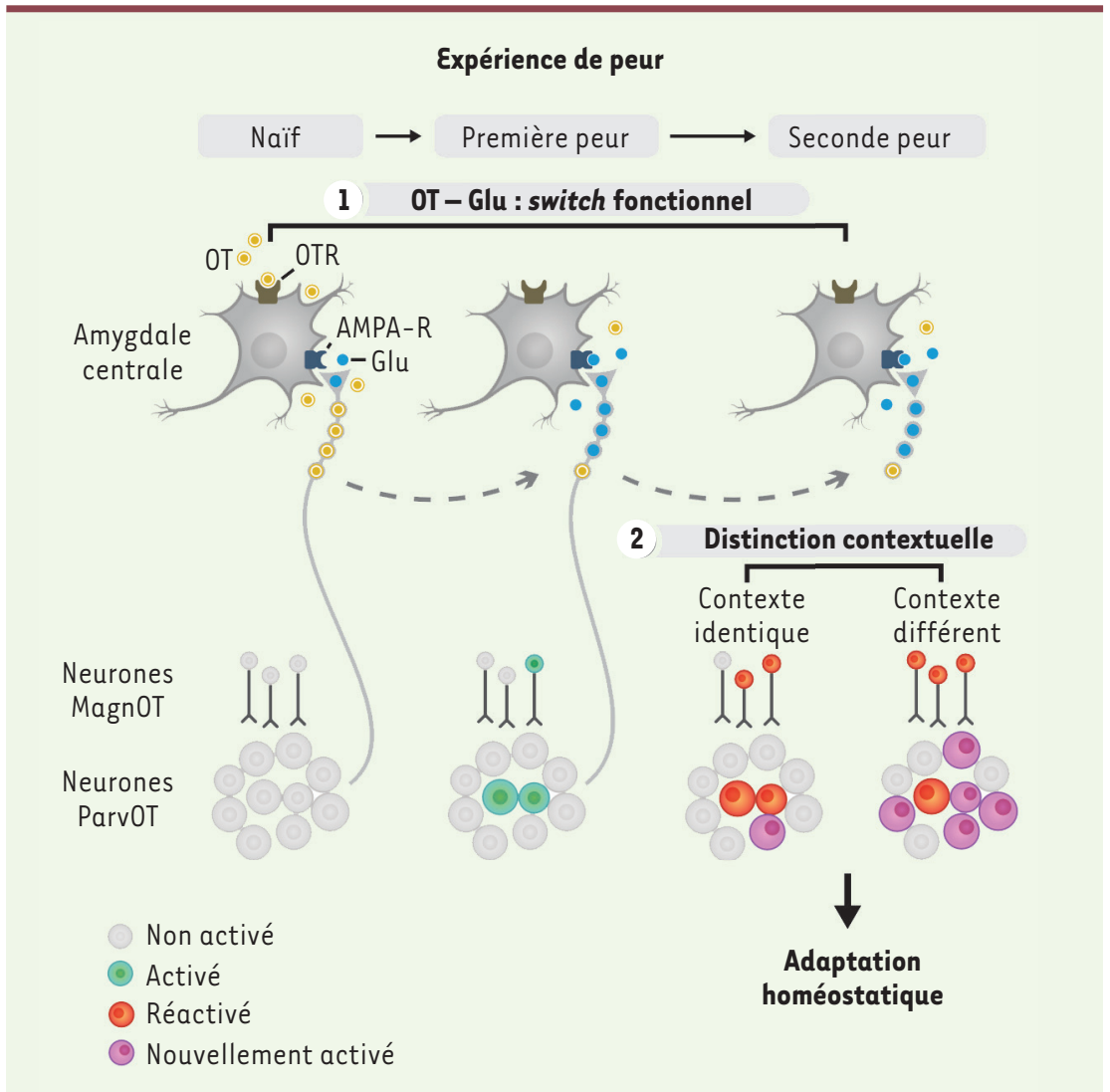

Figure 2. Schéma récapitulatif des expériences présentées. 1. Une expérience de peur chez le rat induit une plasticité neuronale qui se traduit notamment par un switch fonctionnel de neurotransmetteur entre ocytocine (OT) et glutamate (Glu). 2. La propriété de dépendance du contexte de la population des neurones activés atteste de l'existence d'un engramme ocytocinergique. parvOT : neurones parvocellulaires produisant l'ocytocine ; magnOT, neurones magnocellulaires produisant l'ocytocine; AMPA-R : récepteur AMPA du glutamate.

neuronales activées lors de réactions de peur obtenues par l'exposition des rats à différents contextes. Par cette approche, ils ont montré que les assemblées de neurones activés sont spécifiquement associées à un contexte donné. Ainsi, il existe dans l'hypothalamus un engramme constitué de neurones ocytocinergiques permettant d'associer un contexte précis à une émotion de peur (Figure 2).

\section{Un engramme pourvu de plasticité}

On sait que les souvenirs se modifient au fil des expériences vécues. Les chercheurs ont donc voulu savoir si l'engramme ocytocinergique découvert est, à l'image de la mémoire, capable de plasticité. À cette fin, ils ont enregistré l'activité électrophysiologique de neurones de l'amygdale. Les données électrophysiologiques et transcriptomiques obtenues ont permis de montrer que les neurones hypothalamiques libèrent majoritairement de l'ocytocine dans l'amygdale chez les rats « naïfs », c'est-à-dire non exposés au conditionnement de peur (Figure 1-2). Étonnamment, après que l'animal a vécu une première expérience de peur les neurones hypothalamiques libèrent préférentiellement du glutamate (Figure 2). Une telle variation du type de neurotransmetteur libéré permet d'expliquer que la maîtrise de la peur via l'hypothalamus est plus rapide et 
plus importante face à un stimulus déjà rencontré. Cet important changement de neurotransmetteur libéré, ce «switch fonctionnel » de l'ocytocine vers le glutamate, démontre ainsi l'existence d'une véritable plasticité fonctionnelle de cet engramme [2].

Finalement, une peur donnée induit le recrutement d'un groupe de neurones ocytocinergiques de l'hypothalamus permettant le contrôle de cette peur. Par ailleurs, une expérience de peur modifie le fonctionnement de cet engramme en provoquant un changement du neurotransmetteur libéré, de l'ocytocine vers le glutamate. Une telle conversion de la molécule sécrétée est en accord avec la vision selon laquelle les processus cognitifs et émotionnels seraient modulés par un équilibre entre les neuropeptides, dont l'activité neuromodulatrice est lente, et les neurotransmetteurs «classiques» (e.g. glutamate, GABA, acétylcholine), qui ont une activité bien plus brève [8]. Dans ce contexte, l'ocytocine laisse place au glutamate après le conditionnement de peur associée au contexte chez le rat, modifiant ainsi la rapidité de réaction face à un stimulus effrayant et donc potentiellement dangereux, afin de permettre une meilleure adaptation homéostatique (fréquence cardiaque, activité métabolique, etc.) [2].

\section{Cibler cet engramme pour agir sur la} peur pathologique

Hasan et collaborateurs ont donc découvert que des engrammes répondant à tous les critères de la théorie synaptique de la mémoire [9] peuvent se former dans l'hypothalamus. Ils ont ainsi montré pour la première fois que les souvenirs ne sont pas uniquement stockés dans les structures cérébrales dites supérieures (cortex, hippocampe) [10], mais aussi dans des régions apparues beaucoup plus tôt au cours de l'évolution, telles que l'hypothalamus. Cette découverte majeure incite à rechercher l'existence des engrammes dans les différentes régions cérébrales et à en explorer l'intégration. Comprendre les circuits anatomiques et fonctionnels impliqués dans une mémoire émotionnelle telle que celle de la peur pourrait permettre l'émergence de nouvelles stratégies thérapeutiques, notamment quand la peur devient pathologique, comme dans le cas de l'anxiété chronique ou du stress post-traumatique. $\diamond$ An oxytocinergic engram to learn and control our fear
LIENS D'INTÉRÊT

Les auteurs déclarent n'avoir aucun lien d'intérêt concernant les données publiées dans cet article.

\section{RÉFÉRENCES}

1. On memory and reminiscence; written 350 B.C. $\varepsilon$ by Aristote, translated by J.I. Beare. http://classics.mit. edu/Aristotle/memory.html

2. Hasan M, Althammer F, Silva da Gouveia M, et al. A fear memory engram and its plasticity in the hypothalamic oxytocin system. Neuron $2019 ; 103$ : 133-46.

3. Viviani $D$, Charlet $A$, van den Burg $\varepsilon$, et al. Oxytocin selectively gates fear responses through distinct outputs from the central amygdala. Science 2011; 333: 104-7.

4. Dale H. On some physiological actions of ergot.J Physiol 1906 ; 34 : 163-206.

5. Lee H, Macbeth A, Pagani J, Young W. Oxytocin: the great facilitator of life. Prog Neurobiol 2009; 88 127-51

6. Eliava M, Melchior M, Knobloch-Bollmann H, et al. A new population of parvocellular oxytocin neurons controlling magnocellular neuron activity and inflammatory pain processing. Neuron $2016 ; 89$ : 1291-304.

7. Knobloch H, Charlet A, Hoffmann L, et al. Evoked axonal oxytocin release in the central amygdala attenuates fear response. Neuron 2012 ; 73 : 553-66.

8. Merighi A, Salio C, Ferrini F, Lossi L. Neuromodulatory function of neuropeptides in the normal CNS. J Chem Neuroanat $2011 ; 42: 276-87$.

9. Martin S, Grimwood P, Morris R. Synaptic plasticity and memory: an evaluation of the hypothesis. Annu Rev Neurosci $2000 ; 23: 649-711$.

10. Kitamura T, Ogawa S, Roy D, et al. Engrams and circuits crucial for systems consolidation of a memory. Science 2017 ; 356 : 73-8.

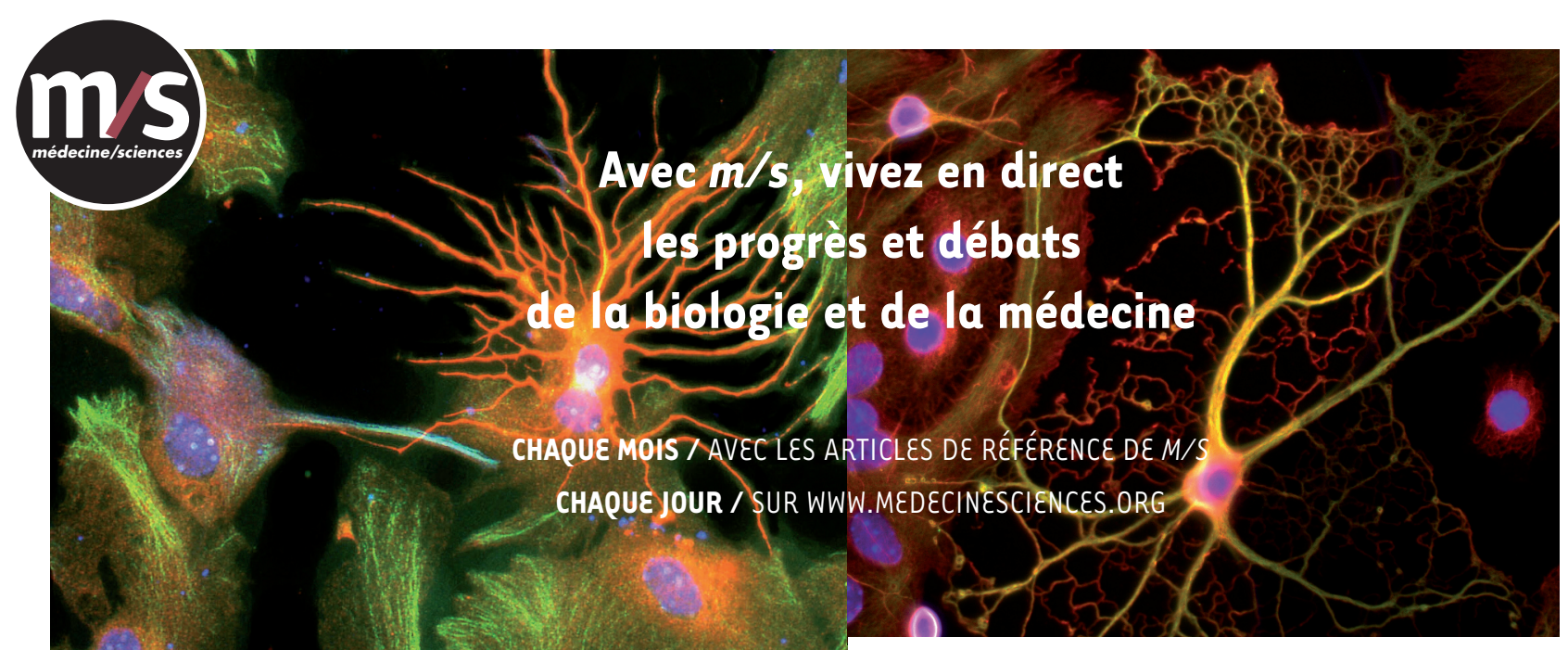

Abonnez-vous sur

www.medecinesciences.org 